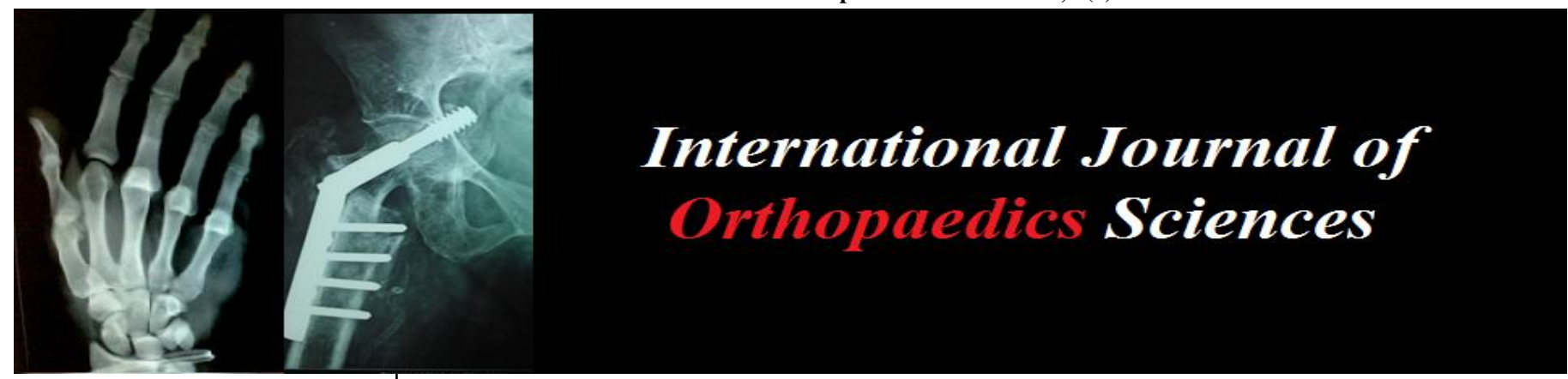

E-ISSN: 2395-1958

P-ISSN: 2706-6630

IJOS 2019; 5(4): 147-151

(C) 2019 IJOS

www.orthopaper.com

Received: 14-11-2019

Accepted: 16-12-2019

Dr. Manas Satpathy Resident NIMS, Jaipur,

Rajasthan, India

Dr. Subhash Jain Associate Professor NIMS,

Jaipur, Rajasthan, India

Dr. Ramgopal Gupta Professor NIMS, Jaipur,

Rajasthan, India

Dr. Shalini Agrawal

Assistant professor GMCH,

Udaipur, Rajasthan, India
Corresponding Author

Dr. Subhash Jain

Associate Professor, NIMS,

Jaipur, Rajasthan, India

\section{Comparative evaluation of results of physiotherapy alone, periarticular injections followed by physical exercise and manipulation under anaesthesia followed by physical exercise in adhesive capsulitis of shoulder}

\author{
Dr. Manas Satpathy, Dr. Subhash Jain, Dr. Ramgopal Gupta and Dr. \\ Shalini Agrawal
}

DOI: https://doi.org/10.22271/ortho.2020.v6.i1c.1851

\section{Abstract}

Introduction: The enigma of Adhesive Capsulitis also referred as 'frozen shoulder' is an inflammatory contracture of the glenohumeral joint capsule, leading to shoulder pain and stiffness. It affects $2-5 \%$ of the population. It has been a challenge to our profession for many decades because of its obscure etiology, varied pathological processes, and improper therapeutic measures.

Objectives: To compare and evaluate the safety, efficacy and results for the treatment of adhesive capsulitis by Physiotherapy, Peri-articular injections followed by physiotherapy and Manipulation under Anaesthesia (MUA) followed by physiotherapy.

Methodology: This Prospective randomized comparative study was conducted on patients with adhesive capsulitis of shoulder admitted in Department of Orthopedics, NIMS, Jaipur for 6 months from $1^{\text {st }}$ January, 2018 to $30^{\text {th }}$ June, 2018. A total of 150 cases (50 in each category) were taken, out of which 30 patients were lost to follow up. Patients were divided into three groups, in first group(A) all patients were managed by Manipulation Under Anaesthesia (MUA) followed by physiotherapy, Second group (B) were treated by periarticular injections of Methyl Prednisolone and Lignocaine(2\%) followed by physiotherapy while in third group (C), by Physiotherapy only.

Results: Adhesive capsulitis was found to be more prevalent among males. 55\% of the patient in our study were Males, peak incidence of adhesive capsulitis between age group of 40 to 70 year. Maximum number of cases were found between 41-50year (50\%) and 51 to 60 year $(38.33 \%)$. Highest no of patients were found as daily wage worker (33) $27.5 \%$ followed by house wife (32) $26.67 \%$. Least patients were found in student class (2)1.67\%. Side of affection was majorly left shoulder. Results were evaluated on the basis of clinical improvement of certain parameters such as Pain, Tenderness, Muscular Atrophy and Range of Movement Of Shoulder. Atrophy of muscle was seen in 71 patients. Atrophy of only Deltoid muscle was observed in 36 patients. Except in 4 patients all pts had tenderness over shoulder. All the treatment modalities were found to be significant with all $\mathrm{p}$ values less than 0.05 . Good grade was observed nearly equivalent in MUA with PT $(80.43 \%)$ and Injection with PT $(78.57 \%)$ with a difference of $1.86 \%$. Poor grade was found maximum in Physiotherapy $(28.12 \%)$ and minimum in MUA with PT $(4.35 \%)$.

Conclusion: The study conclude MUA with PT and Inj with PT showed nearly equivalent good results. Physiotherapy alone showed maximum poor results. This study supports the use of Manipulation under Anaesthesia with PT and Peri-articular injection with PT over Physiotherapy alone.

Keywords: adhesive capsulitis, manipulation under anaesthesia (MUA), methyl prednisolone

\section{Introduction}

Adhesive Capsulitis (Frogen Shoulder) is an inflammatory contracture of the glenohumeral joint capsule, leading to shoulder pain and stiffness especially in external rotation and abduction. It is believed that inflammation happened in rotator interval, results in painful motion and subsequent fibrosis, and stiffness that limits movements.

It affects $2-5 \%$ of the population, commonly involving women between the ages 40 to 60 year ${ }^{[4,5]}$ No definitive etiology for it has been established so far.

Frozen Shoulder (FS) can be sub classified as: primary \& secondary conditions. Secondary FS 
(SFS) is allied with a defined event, such as a known intrinsic (rotator cuff pathology, osteoarthritis) or extrinsic (trauma) cause whereas Primary FS (PFS) is differentiated by an insidious onset of idiopathic origin FS associated with medical conditions such as Diabetes mellitus or with thyroid diseases are categorized as secondary systemic frozen shoulder.

Table 1: Clinical and histopathological stages of adhesive capsulitis

\begin{tabular}{|c|c|c|c|}
\hline Stages & Symptoms & Length of symptoms & Histological features \\
\hline Painful phase & Aching pain and moderate limitation of ROM & Less than 3 months & Synovitis and capsular hypertrophy \\
\hline Freezing stage & Severe pain and reduction of ROM & 3 to 9 months & Intravascular synovitis disorganized collagen deposition \\
\hline Frozen stage & Stiffness is predominant. Pain may be present & 9 to 14 months & Dense and hypercellular collagenous tissue \\
\hline Thawing stage & Minimal pain and a gradual improvement of ROM & 15 and 24 months & Not investigated \\
\hline
\end{tabular}

\section{Adhesive capsulitis has 4 phases of progression}

1) Painful Phase-less than 3 months: Aching pain

2) Initial (Freezing stage)-2 to 9 months : diffuse, severe pain at night

3) Intermediate (Frozen Stage)-4 to 12 months: stiffness and decreased range of motion with less pain.

4) Recovery (Thawing Stage)-5 to 24 months: gradual return of range of motion and resolution of pain

Symptoms that are associated with frozen shoulder are: pain with movement, localized pain, nocturnal pain, restriction of active and passive ROM (especially external rotation) and radiographic imaging are found to be normal findings ${ }^{13}$.

The absence of conclusive diagnostic criteria has imposed challenges for the clinical diagnosis, management and research of adhesive shoulder capsulitis, but helps to rule out other aetiology of stiff shoulder such as:, calcific tendonitis, rotator cuff tendinopathy or early glenohumeral dislocation and humeral fracture.

There are many methods of treatment for this disorder including physiotherapy, manipulation under anaesthesia with physical exercise, peri-articular injections with physical exercise, arthroscopic dilatation of the joint and surgery.

\section{Objectives}

To compare and evaluate the safety, efficacy and results for the treatment of adhesive capsulitis by Physiotherapy, Periarticular injections followed by physiotherapy and Manipulation under Anaesthesia (MUA) followed by physiotherapy.

\section{Material and Methods}

This Prospective randomized comparative study was conducted in Department of Orthopedics, National Institute of Medical Sciences and Research, Jaipur for 6 months from $1^{\text {st }}$ January, 2018 to $30^{\text {th }}$ June, 2018. Study included 150 patients with adhesive capsulitis of shoulder fulfilling the inclusion and exclusion criteria.

\section{Inclusion criteria}

- Patients with pain and stiffness of shoulder without any other pathology.

- Age between 40-70 years

- No previous trauma or surgery to shoulder

- Patients given consent to participate in my study

\section{Exclusion criteria}

- Previous major trauma

- Patients with Rheumatoid Arthritis

- Patients with Locked Anterior and Posterior dislocation of shoulder

- Patients with Subacromial Impingement Syndrome

- Patients with Rotator cuff lesion

- Patients with Diabetes Mellitus

- Infection of Shoulder and previous shoulder surgery

- Any form of coagulopathy

- Age $<40$ and $>70$ year

\section{Methodology}

150 patients of adhesive capsulitis of shoulder diagnose clinically during the study period were taken, out of which 30 patients were lost to follow up. Hence, 120 patients were enrolled. 46 patients were enrolled in MUA f/b PT, 42 patients in Peri-articular inj. f/b PT and 32 in PT group.

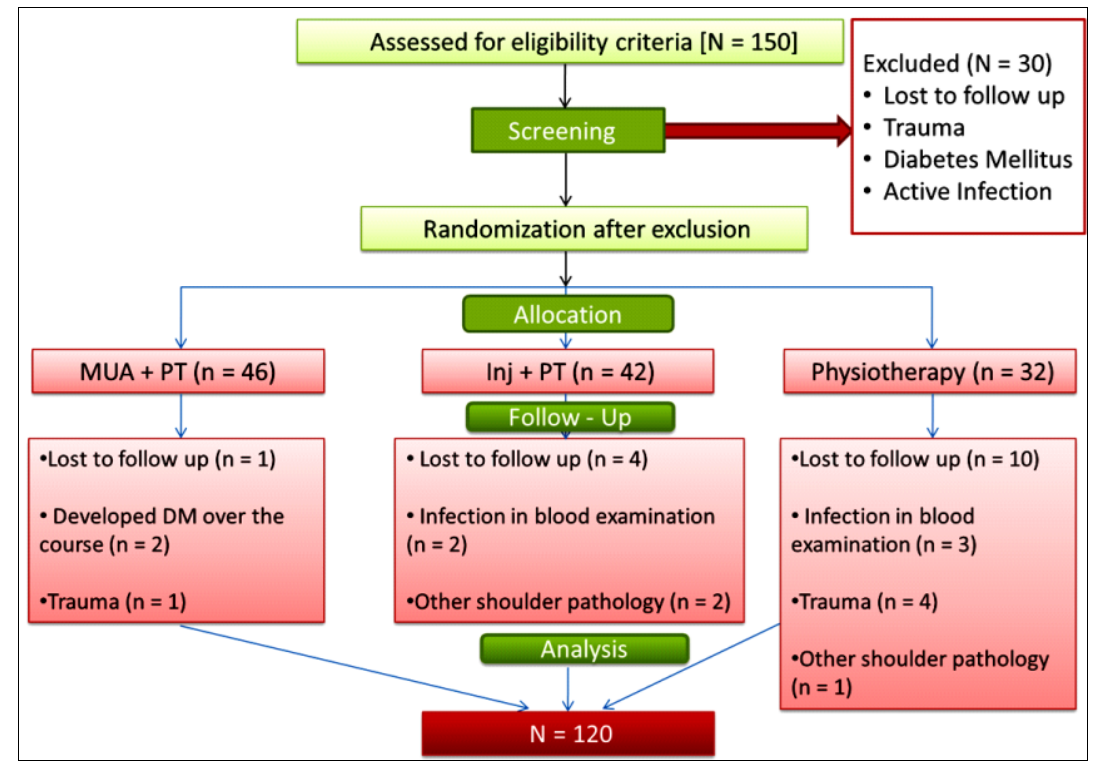

Fig 1: Division of patients after admission 
The patients were divided into three groups based on the treatment

1) First group (A) were treated with Manipulation Under Anaesthesia (MUA) followed by physiotherapy

2) Second group (B) were treated with local periarticular injections of Methyl Prednisolone and Lignocaine (2\%) followed by physiotherapy

3) Third group (C) were treated with Physiotherapy only The patients were called for follow up twice during the study period at an interval of 3 months.

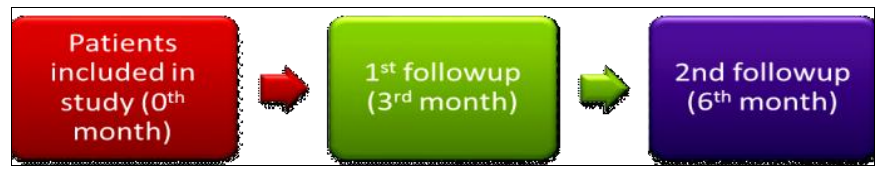

Fig 2: Timeline of follow up of patients

\section{1) Physiotherapy}

The patients were treated in the department of physical medicine on OPD basis. Following methods were used-Active and passive shoulder mobilization exercises, Shoulder wheel and pulley exercises, Ultra Sound therapy, Short wave diathermy, Electrical stimulation and Interferential Therapy (IFT)

\section{2) Periarticular injection followed by physical therapy}

In all cases proper aseptic precaution were taken, and the site of injection was ascertained by anatomical landmarks and marked by skin pencil. Subsequently Methyl Prednisolone injection (Depo-Medrol 40mg/ml + Lignocaine 2\%) were given, using the posterior approach. Analgesics were given post injection period for 2 to 3 days and physical therapy was started 3 to 4 days after injection till a period of 6 months.

\section{3) Manipulation under anesthesia followed by physical therapy}

After general anaesthesia or interscalene block manipulations was done in the sequence of (FEAR) Flexion, Extension, Abduction, Adduction, external Rotation and internal Rotation. Analgesics were given post manipulation period for 2 to 3 days and physical therapy was started 3 to 4 days after manipulation till 6 months.

\section{Evolution of results}

Results were evaluated on the basis of clinical improvement of certain parameters such as Pain, Tenderness, Muscular Atrophy and Range of Movement of Shoulder in Different Planes

The clinical results were graded as below for quick review Good: No pain, no tenderness present, ROM is equal or comparable with normal limb, muscular atrophy recouped.

Fair: Mild pain and tenderness may or may not be present, Mild restriction of ROM still present even after 6 months, muscular atrophy may or may not be present.

Poor: Gross restriction of movement still present with pain, moderate to severe tenderness with muscular atrophy.
Results

Table 2: Categorization Based on Age

\begin{tabular}{|c|c|}
\hline Age (in years) & No. of patients \\
\hline 40 & 6 \\
\hline $41-50$ & 60 \\
\hline $51-60$ & 46 \\
\hline $61-70$ & 8 \\
\hline
\end{tabular}

Age group with maximum prevalence was 41-60. Majority of the patients $50 \%$ were in the age group of $41-50$ followed by $38.33 \%$ in the age group 51-60, 6.6\% in the age group 61-70.

Table 3: Categorization based on gender

\begin{tabular}{|c|c|}
\hline Male & Female \\
\hline 66 & 54 \\
\hline
\end{tabular}

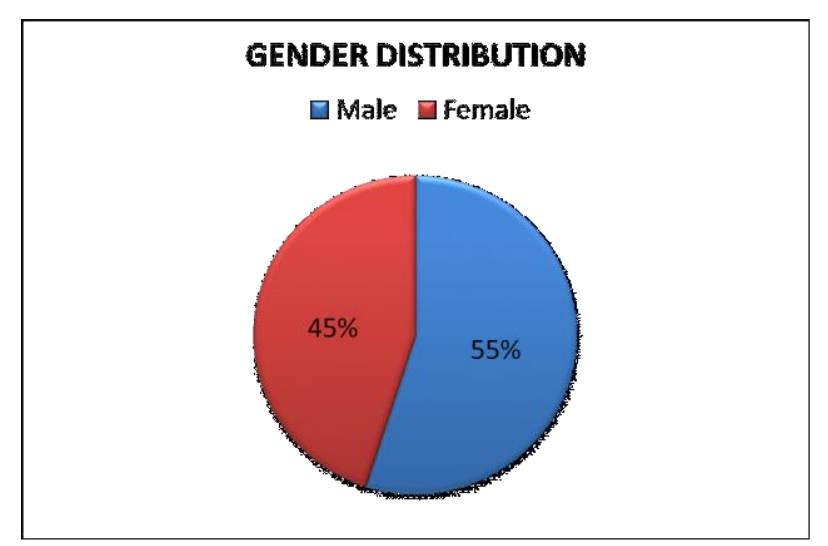

Fig 3: Gender distribution in the study population

Adhesive capsulitis was found to be more prevalent among males. $55 \%$ of the patient in our study were male while female patients constituted $45 \%$ of the study population.

Table 4: Characterization based on occupational status

\begin{tabular}{|c|c|c|}
\hline Occupation & No. of patients & Percentage \\
\hline Daily wage worker & 33 & $27.5 \%$ \\
\hline Govt. job & 13 & $10.83 \%$ \\
\hline House wife & 32 & $26.67 \%$ \\
\hline Private job & 3 & $2.5 \%$ \\
\hline Student & 2 & $1.67 \%$ \\
\hline Farmer & 23 & $19.16 \%$ \\
\hline Business & 14 & $11.67 \%$ \\
\hline
\end{tabular}

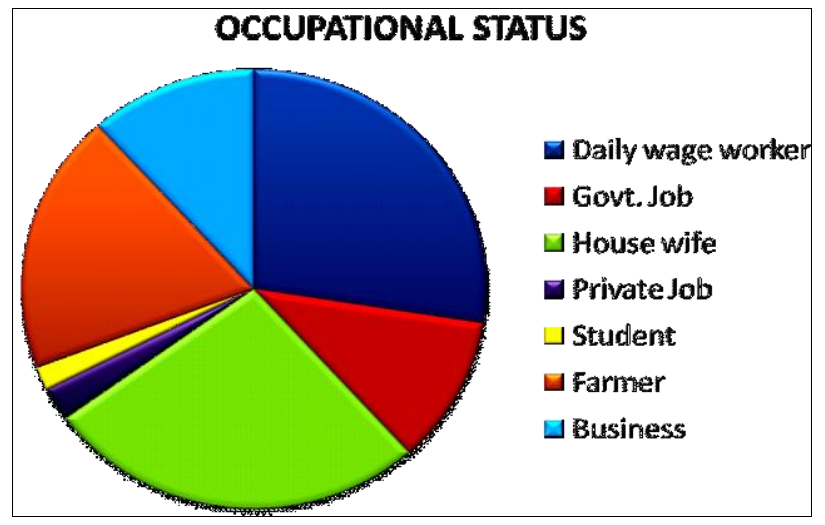

Fig 4: Occupational status of study population 
Highest no of patients were found as daily wage worker (33) $27.5 \%$ followed by house wife (32)26.67\% and farmers (23) $19.16 \%$ consecutively. Least rate of patients were found in student class (2) $1.67 \%$.

Table 5: Characterization based on side of affection

\begin{tabular}{|c|c|}
\hline Right Shoulder & Left Shoulder \\
\hline 57 & 63 \\
\hline
\end{tabular}

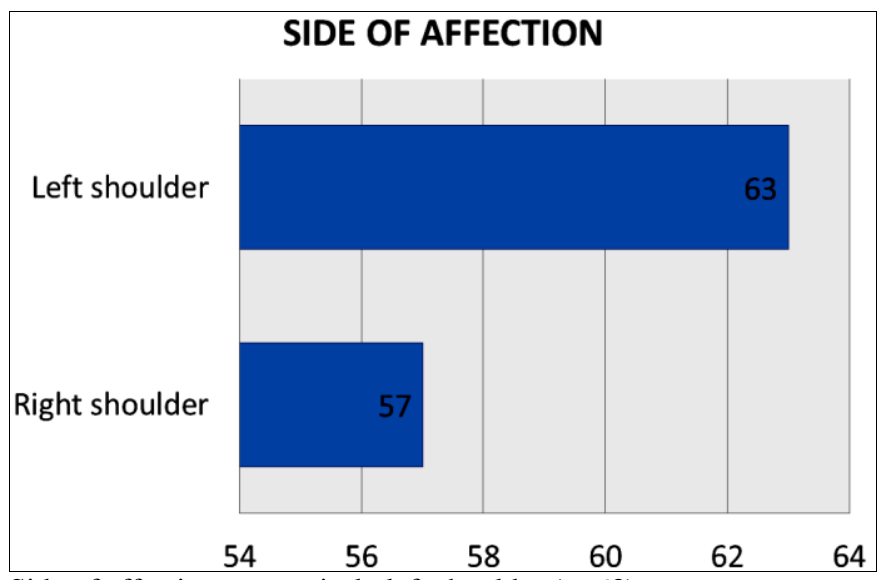

Side of affection was majorly left shoulder $(n=63)$

Fig 5: Side of affection in study population

These grading are based on patient's condition after 6 months of treatment.

Table 6: Comparison of Grading of all three treatment modalities

\begin{tabular}{|c|c|c|c|c|}
\hline Treatments & Good & Fair & Poor & P Value \\
\hline MUA + PT $(n=46)$ & 37 & 7 & 2 & 0.015 \\
\hline Inj + PT $(n=42)$ & 33 & 6 & 3 & 0.03 \\
\hline Physio & 11 & 9 & 12 & 0.05 \\
\hline
\end{tabular}

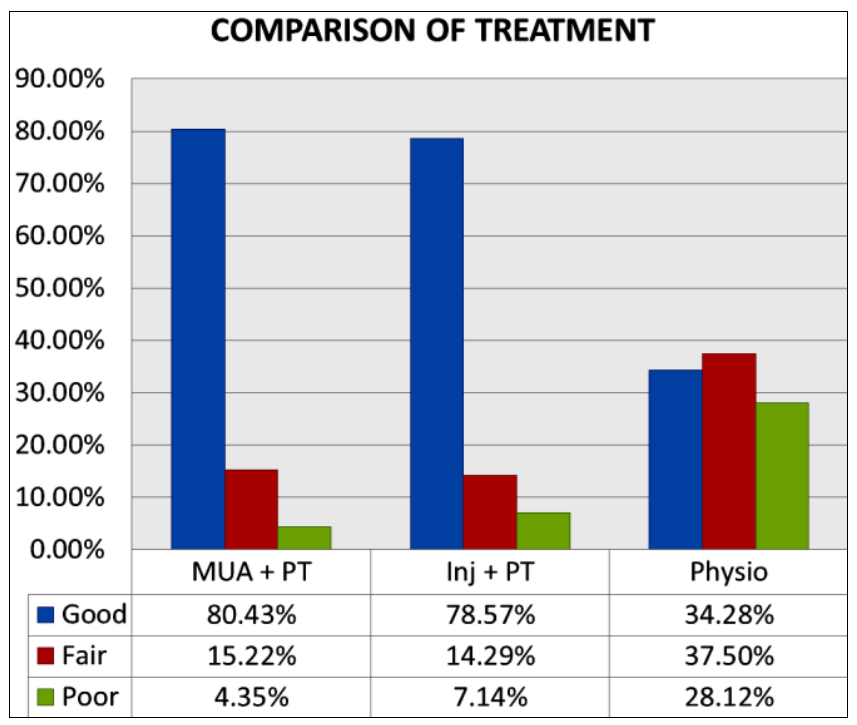

Fig 6: Comparison between all three treatment modalities with grades

All the treatment modalities were found to be significant with all $\mathrm{p}$ values less than 0.05 . In the following series, Good grade was observed nearly equivalent in MUA with PT $(80.43 \%)$ and Injection with PT $(78.57 \%)$ with a difference of $1.86 \%$. Fair grade was observed in MUA with PT (15.22\%), Inj. Depo-medrol with PT (14.29\%). Fair grade difference was $0.93 \%$. In Physiotherapy, the good grade $(34.28 \%)$ and fair grade $(37.50 \%)$ was nearly equivalent with a difference of
$3.22 \%$. Poor grade was found maximum in Physiotherapy $(28.12 \%)$ and minimum in MUA with PT (4.35\%).

\section{Discussion}

Adhesive capsulitis is one of the leading cause of significant morbidity worldwide. Adhesive capsulitis affect the structural and mechanical integrity of the joint. Consequently the smooth gliding action of the joint and its range of movements are lost which is associated with pain. The primary aim in the treatment of adhesive capsulitis is painless shoulder with reasonably good range of motion both active and passive. Pain was a constant feature in almost all cases. The pain is usually felt about the shoulder or near the insertion of the deltoid. Following treatment the intensity of pain was gradually diminished.

Adhesive capsulitis is described as a pathological process in which body form excessive scar tissue or adhesion across the glenohumeral joint which lead to stiffness, pain and impaired movement of shoulder. (Hai V. Le) ${ }^{[23]}$.

It is having incident of $2 \%$ to $5 \%$ in general population. Kingston et al. ${ }^{[24]}$ shows the peak incidence of adhesive capsulitis between age group of 40 to 70 year. In our study Age group with maximum prevalence was 41-60. Majority of the patients $50 \%$ were in the age group of $41-50$ followed by $38.33 \%$ in the age group 51-60, 6.6\% in the age group 61-70. Muscular atrophy mainly of deltoid muscle was found in about $60 \%$ case. Atrophy of muscle was seen in 71 patients. Atrophy of only Deltoid muscle was observed in 36 patients. But in other cases either atrophy of supraspinatus 24 or of Suraspinatus and infraspinatus 11 found. Except in 4 patients all pts had tenderness over shoulder either in anterior aspect or all over the shoulder.

Imran Butt et al. shows the ratio of female to male was 1:3 and in our study male $(55 \%)$ and female $(45 \%)$ but few studies are reported to have male and female ratio 1:1 Bunker et al. ${ }^{[25]}$ Regarding affected shoulder right $(n=76)$, left $(n=69)$ whereas in our study affected shoulder right $(n=57)$, left $(n=63)$.

Imran Butt et al. result shows no significant difference between intra-articular steroids $(2.2571 \pm 0.7808$, p value - < $0.001)$ and MUA $(2.2541 \pm 0.7808, \mathrm{p}$ value $-<0.001)$ but they concluded Intra articular injection methods is more preferable over MUA.

\section{Conclusion}

Adhesive capsulitis is a specific condition that has a natural history of spontaneous resolution and requires management pathway that is completely different from such distinct shoulder conditions as a rotator cuff tear or osteoarthritis.

The study conclude Manipulation under Anaesthesia with physical therapy showed maximum Good grade followed by Peri-articular injection with physical therapy and Physiotherapy in results. MUA with PT showed nearly equivalent results to Inj with PT. Physiotherapy alone showed maximum poor grades among the 3 therapies.

Thus, this study supports the use of MUA with PT and Periarticular injection with PT over Physiotherapy alone.

\section{References}

1. Codman EA. The shoulder Boston: Thomas Todd Co, 1934.

2. Neviaser IS. Adhesive capsulitis of the shoulder: a study of the pathological findings in periarthritis of the shoulder. J Bone Joint Surg. 1945; 27:211-22.

3. Yaying Sun MSL. Steroid Injection versus Physiotherapy 
for Patients with Adhesive Capsulitis of the Shoulder. MD-Journal, 2016, 95.

4. Tim Kraal RB. Manipulation under anesthesia versus physiotherapy treatment in stage two of a frozen shoulder: a study protocol for a randomized controlled trial. BMC Musculoskeletal Disorders, 2017, 412(18).

5. Lho YM, Ha E, Cho $\mathrm{CH}$ et al. Inflammatory cytokines are overexpressed in the subacromial bursa of frozen shoulder. J Shoulder Elbow Surg. 2013; 22:666-672.

6. Shah N, Lewis M. Shoulder adhesive capsulitis: systematic review of randomised trials using multiple corticosteroid injections. Br J Gen Pract. 2007; 57:662667.

7. Lippmann RK. Frozen shoulder, periarthritis, bicipital tenosynovitis, Arch Surg. 1943; 47:283.

8. Lundberg BJ. The frozen shoulder. Acta Orthop Scand Suppl. 1969; 119:1

9. Hazleman BL. The painful stiff shoulder. Rheumatol Phys Med. 1972; 11:413-21.

10. Green S, Buchbinder R, Glazier R et al. Systematic review of randomized controlled trails of interventions for painful shoulder: selection criteria, outcome assessment, and efficacy. BMJ. 1998; 316:354-60

11. Neviaser RJ, Neviaser TJ. The frozen shoulder: diagnosis and management. Clin Orthop Relat Res. 1987; 223:5964.

12. Reeves B. The natural history of the frozen shoulder syndrome. Scand J Rheumatol. 1975; 4:193-196.

13. Zuckerman JD, Rokito A et al. Frozen shoulder: a consensus definition. J Shoulder Elbow Surgery. 2011; 20(2):322-5. Doi:10.1016/j.jse.2010.07.008.

14. Milgrom C, Novack V, Weil Y, Jaber S, Radeva-Petrova DR, Finestone A et al. Risk factors for idiopathic frozen shoulder. Israel Med Assoc J. 2008; 10(5):361-4.

15. Sofka CM, Ciavarra GA, Hannafin JA, Cordasco FA, Potter HG. Magnetic resonance imaging of adhesive capsulitis: correlation with clinical staging. HSS J. 2008; 4(2):164-9. Doi:10.1007/s11420-008-9088-1.

16. Hand GC, Athanasou NA, Matthews T, Carr AJ. The pathology of frozen shoulder. J Bone Joint Surg. 2007; 89(7):928-32. Doi:10.1302/0301-620X. 89B7.19097.

17. Jacobs LGH, Barton MAJ, Wallace WA et al. intraarticular distension and steroids in the management of capsulitis of the shoulder. BMJ. 1991; 302:1498-1501 [Medline].

18. Oglivie-Harris DJ, Biggs DJ, Fitsialos DP, Mackay M. The resistant frozen shoulder: manipulation versus arthroscopic release, Clin Ortho. 1995; 319:238.

19. Gram AN, Schydlowsky P, Rossel I et al. Treatment of frozen shoulder, with distension and glucocorticoid campared with glucocorticoid alone. Scand J Rheumatol. 1998; 27:425-30 [Cross Ref][Medline].

20. Cartte S, Moffet $\mathrm{H}$, Tardiff $\mathrm{J}$ et al. Intraarticular corticosteriods, supervised physiotherapy, or a combination of the two in the treatment of adhesive capsulitis of the shoulder. Arthritis Rheum. 2003; 48:82938 [Cross Ref] [Medline].

21. Buchbinder R, Green S, Forbes A et al. Arthrographic joint distension with saline and steroid improves function and reduces pain in patients with painful stiff shoulder: results of a randomized, ouble-blind, placebo-controlled trial. Ann Rheum dis. 2004; 63:302-9.

22. Muhammad IB, Tooba I. Comparison between manipulation under anaesthesia and intra-articular steroid injection for frozen shoulder; Journal of Rawalpindi
Medical College. 2018; 22(4):342-345.

23. Hai Le V, Stekka JL et al. Adhesive capsulitis of shoulder: review of pathophysiology and current clinical treatment. Journal of shoulder and elbow. 2017: 9(2)758423.

24. Kingson K, Emily J et al. Shoulder adhesive Capsulitis: Epidemiology and predictor of surgery. Journal of shoulder and elbow. 2018; 04:09.

25. Bunker TD, Reilly J, Baird KS et al. Expression of growth factors, cytokines and matrix metalloproteinases in frozen shoulder. J Bone Joint Surg Br. 2000; 82:768773. 